\title{
Interdependence of photon upconversion performance and antisolvent processing in thin-film halide perovskite-sensitized triplet-triplet annihilators
}

Cite as: J. Chem. Phys. 153, 164711 (2020); https://doi.org/10.1063/5.0026564

Submitted: 24 August 2020 . Accepted: 12 October 2020 . Published Online: 28 October 2020

(D) Karunanantharajah Prashanthan, (D) Boris Naydenov, (D) Klaus Lips, (D) Eva Unger, and (iD) Rowan w. MacQueen

\section{COLLECTIONS}

Paper published as part of the special topic on Up- and Down-Conversion in Molecules and Materials
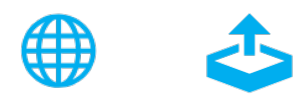

\section{ARTICLES YOU MAY BE INTERESTED IN}

Perovskite-sensitized upconversion bingo: Stoichiometry, composition, solvent, or temperature?

The Journal of Chemical Physics 153, 084703 (2020); https://doi.org/10.1063/5.0021973

Sensitizer-host-annihilator ternary-cascaded triplet energy landscape for efficient photon upconversion in the solid state

The Journal of Chemical Physics 153, 161102 (2020); https://doi.org/10.1063/5.0025438

Red-to-blue photon upconversion based on a triplet energy transfer process not retarded but enabled by shell-coated quantum dots

The Journal of Chemical Physics 153, 114701 (2020); https://doi.org/10.1063/5.0023052

Challenge us.

What are your needs for periodic signal detection?

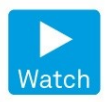

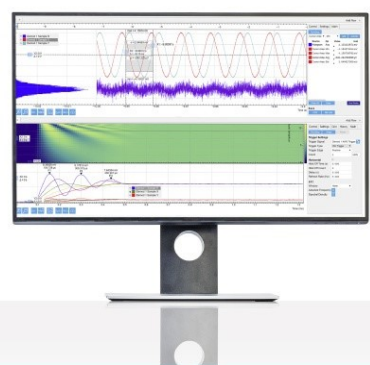

Zurich - Instruments 


\title{
Interdependence of photon upconversion performance and antisolvent processing in thin-film halide perovskite-sensitized triplet-triplet annihilators
}

\author{
Cite as: J. Chem. Phys. 153, 164711 (2020); doi: 10.1063/5.0026564
}

Submitted: 24 August 2020 - Accepted: 12 October 2020 •

Published Online: 28 October 2020

\section{Ð 1}

Karunanantharajah Prashanthan, ${ }^{1,2}$ (D) Boris Naydenov, ${ }^{1}$ (D) Klaus Lips, ${ }^{1}$ (D) Eva Unger, and Rowan W. MacQueen ${ }^{1, a)}$ (iD)

\author{
AFFILIATIONS \\ ${ }^{1}$ Department Spins in Energy Conversion and Quantum Information Science (EE-ASPIN), Helmholtz-Zentrum Berlin für \\ Materialien und Energie $\mathrm{GmbH}$, Berlin, Germany \\ ${ }^{2}$ Department of Physics, University of Jaffna, Jaffna, Sri Lanka \\ ${ }^{3}$ Young Investigator Group Hybrid Materials Formation and Scaling, Helmholtz-Zentrum Berlin fur Materialien und Energie \\ GmbH, Berlin, Germany \\ ${ }^{4}$ Chemical Physics and NanoLund, Lund University, Lund, Sweden
}

Note: This paper is part of the JCP Special Topic on Up- and Down-Conversion in Molecules and Materials.

a) Author to whom correspondence should be addressed: rowan.macqueen@helmholtz-berlin.de

\begin{abstract}
We prepared triplet-triplet annihilation photon upconverters combining thin-film methylammonium lead iodide (MAPI) perovskite with a rubrene annihilator in a bilayer structure. Excitation of the perovskite film leads to delayed, upconverted photoluminescence emitted from the annihilator layer, with triplet excitation of the rubrene being driven by carriers excited in the perovskite layer. To better understand the connections between the semiconductor properties of the perovskite film and the upconversion efficiency, we deliberately varied the perovskite film properties by modifying two spin-coating conditions, namely, the choice of antisolvent and the antisolvent dripping time, and then studied the resulting photon upconversion performance with a standard annihilator layer. A stronger upconversion effect was exhibited when the perovskite films displayed brighter and more uniform photoluminescence. Both properties were sensitive to the antisolvent dripping time and were maximized for a dripping time of $20 \mathrm{~s}$ (measured relative to the end of the spin-coating program). Surprisingly, the choice of antisolvent had a significant effect on the upconversion performance, with anisole-treated films yielding on average a tenfold increase in upconversion intensity compared to the chlorobenzene-treated equivalent. This performance difference was correlated with the carrier lifetime in the perovskite film, which was $52 \mathrm{~ns}$ and $306 \mathrm{~ns}$ in the brightest chlorobenzene and anisole-treated films, respectively. Since the bulk properties of the anisole- and chlorobenzene-treated films were virtually identical, we concluded that differences in the defect density at the MAPI/rubrene interface, linked to the choice of antisolvent, must be responsible for the differing upconversion performance.
\end{abstract}

Published under license by AIP Publishing. https://doi.org/10.1063/5.0026564

\section{INTRODUCTION}

Photon upconversion by triplet-triplet annihilation (TTA) allows the generation of substantially anti-Stokes shifted photoluminescence (PL) from incoherent excitation sources and is of high technological interest for photovoltaics, phototherapy, imaging, and photocatalysis applications. ${ }^{1-4}$ Sensitized TTA-upconversion begins with light absorption by triplet sensitizers and then the generation, via energy transfer, of triplet excitons in a second species known as the annihilator. Annihilators are molecules with electronic 
energy levels that permit TTA - the collision and fusion of two triplet excitons-with a high likelihood of yielding a bright spin-singlet excited state in one of the participating molecules. Relaxation of the singlet-excited annihilator to the ground state by photoluminescence or energy transfer produces the eponymous photon upconversion effect.

Thin-film lead halide perovskites are emerging as a fascinating new component of TTA photon upconversion systems. Configured in a bilayer structure, consisting of the thin-film perovskite interfaced with a layer of a suitable annihilator, TTA-driven photon upconversion in the annihilator occurs following photoexcitation of the perovskite film. ${ }^{5}$ In the terminology of the photon upconversion field, the perovskite film acts as the triplet sensitizer, absorbing low-energy photons and inducing triplet excitations in the annihilator film, as illustrated in Fig. 1. However, the process by which this happens differs considerably from typical sensitizers.

Triplet sensitizers such as thermally activated delayed fluorescence (TADF) molecules, ${ }^{6,7}$ nanocrystals composed of metal chalcogenides, ${ }^{8-11}$ silicon, ${ }^{12}$ or halide perovskites, ${ }^{13}$ transition metal complexes, ${ }^{14}$ and other varieties of metal-free molecular sensitizers ${ }^{15}$ are excitonic, with electronic states defined largely on a single particle basis and with inter-particle effects playing a subordinate role. On the other hand, thin-film halide perovskites are three-dimensional semiconductors, and the excited state processes are correspondingly described in terms of delocalized charge carriers and chemical potential, rather than exciton hopping and electronic energy transfer. Due to their delocalization and high mobility, charge carriers are highly sensitive to defects, structural imperfections within the film

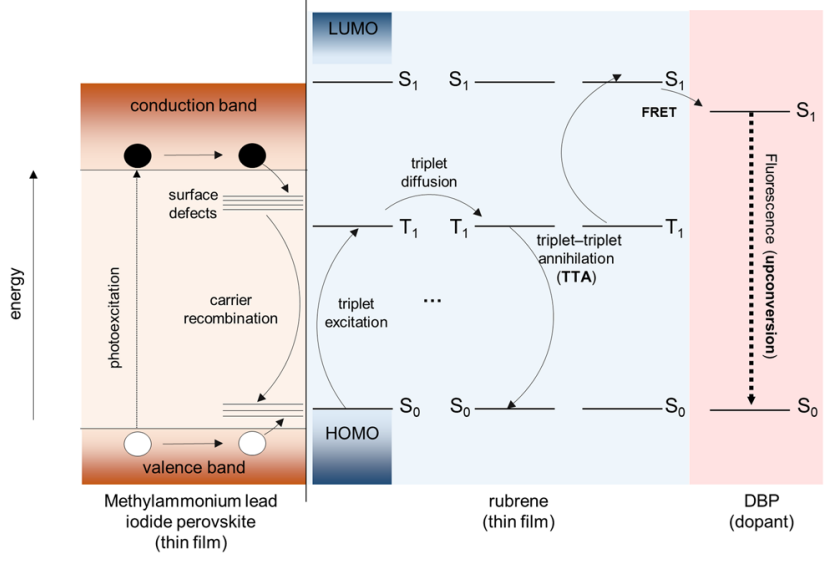

FIG. 1. Scheme of the triplet-triplet annihilation upconversion process operating in a bilayer system consisting of $\sim 500 \mathrm{~nm}$ thick methylammonium lead iodide perovskite below a layer of rubrene $(5,6,11,12$-tetraphenylnaphthacene) doped with DBP (tetraphenyldibenzoperiflanthene) and encapsulated in nitrogen. Electrons (filled circles) and holes (empty circles) excited in the perovskite layer drive triplet formation in the rubrene layer through a process that is as-yet unidentified, but in which the involvement of surface states is strongly implicated (vide infra). Once formed, triplets diffuse throughout the rubrene layer and undergo bimolecular encounters, leading to triplet-triplet annihilation (TTA). The rubrene $\mathrm{S}_{1}$ state formed upon successful TTA can be depopulated by Förster resonance energy transfer (FRET) to a nearby DBP molecule, which then emits upconverted photoluminescence. or at film surfaces that lead to carrier capture and recombination. Recent studies from the perovskite photovoltaics field show that surface defects affect carrier lifetime and quasi-Fermi level splitting to an equal or greater extent than defects in the bulk. ${ }^{16-19}$ Complicating the study of surface defects and their role in the perovskite-sensitized TTA process is the fact that surface defects can be modified by treating perovskite films with small organic molecules ${ }^{16,20}$ including rubrene, ${ }^{21,22}$ a common TTA annihilator.

The mechanism by which triplet exciton formation in the annihilator layer is promoted by photocarriers in the perovskite film is not yet clear. One possibility is sequential charge transfer across the perovskite/annihilator interface. ${ }^{5}$ Here, a photo-induced hole transfer from the perovskite, which depopulates the rubrene highest occupied molecular orbital (HOMO), is followed by an electron transfer into the rubrene lowest occupied molecular orbital (LUMO), with the energy of the resulting triplet excited state lowered by spinpairing of the two electrons left residing in the rubrene frontier orbitals. This mechanism has been proposed to explain triplet formation in a GaAs/copper phthalocyanine bilayer system. ${ }^{23}$ On the other hand, there have also been a number of reports of excitonic triplet energy transfer occurring in lower-dimensional perovskite systems, comprising small organic molecules interleaved with planes of lead halide octahedra. ${ }^{24-26}$ These reports are not necessarily reflective of processes operating in the $3 \mathrm{D}$ perovskite case, since the exciton binding energy rises, and the importance of free carrier processes diminishes as the perovskite dimensionality is reduced. ${ }^{27}$ However, given that the triplet formation process is presumably localized to a $2 \mathrm{D}$ surface, the possible involvement of a $2 \mathrm{D}$ perovskite phase in the triplet formation is worth considering.

Compared to an excitonic sensitizer, the bulk semiconductor characteristics, defect-sensitivity, and generally low photostability of thin film perovskites create new challenges for the preparation and characterization of photon upconverters based on this material. On the other hand, the material holds great potential for eliminating the sensitivity of TTA-upconversion performance to ineffectual triplet exciton diffusion through the light-absorbing medium. The Nienhaus group has been a driver of progress in this area, reporting the first observation of TTA-upconversion from a perovskite/annihilator bilayer system, ${ }^{5}$ the effects of varying the perovskite film thickness, ${ }^{28}$ a new reversible photodegradation pathway, ${ }^{29}$ the role of tetraphenyldibenzoperiflanthene (DBP)-doping of rubrene in the perovskite/annihilator bilayer, ${ }^{30}$ and the effects of tuning the processing conditions. ${ }^{31,32}$ Our work builds on this, examining the connections between perovskite-sensitized TTAupconversion performance, the perovskite film characteristics, and the renowned sensitivity of these to the experimental film fabrication processes. $^{3}$

We elected to study the upconversion performance of a series of perovskite films produced while deliberately varying the film fabrication processes. Using the common halide perovskite methylammonium lead iodide (MAPI) produced via an antisolvent (AS)-assisted spin-coating process, we varied both the antisolvent system and the antisolvent dripping time, deliberately detuning the film formation from a pre-established recipe.

In antisolvent-assisted perovskite film growth, the antisolvent treatment plays a decisive role, inducing the formation of a closed perovskite film with desirable optoelectronic properties. Correctly timed application of the antisolvent occurs during a critical phase 
of the spin-coating process, in which the precursor concentration approaches supersaturation, but spontaneous nucleation has not yet begun. The antisolvent initiates rapid and uniform nucleation across the substrate surface and may form intermediate phases that stabilize key compounds and inhibit the generation of unwanted phases. ${ }^{34}$ The effects of antisolvent processing have been connected to virtually all aspects of halide perovskite optoelectronic performance; among them are device stability, defect density, efficiency, and hysteresis effects. ${ }^{34-36}$ Here, we assess the flow-on effects of antisolvent processing on the perovskite-sensitized TTA-upconversion intensity, using a standard rubrene thin film doped with DBP (tetraphenyldibenzoperiflanthene) as the annihilator in all systems. In this two-component annihilator film, rubrene acts as the tripletbearing host and the TTA center. The DBP is a fluorescent guest molecule that effectively increases the fluorescence quantum yield of the system.

We recorded spatially resolved maps of MAPI photoluminescence and upconversion signals for each film composition at a range of excitation energies, and time-resolved photoluminescence transients for a subset of samples. Our study revealed that the MAPI film characteristics, as gauged by photoluminescence uniformity, brightness, and the carrier lifetime, are strongly connected to the upconversion yield. We focused, in particular, on two samples that differed only in the antisolvent selection and that showed virtually identical bulk properties but substantially different upconversion outputs. We concluded that changes in the MAPI/rubrene interface resulting from the different antisolvent treatments were the likely source of this substantial difference in upconversion performance, in agreement with recent work of Bieber et al. ${ }^{31}$ These findings suggest that interface defects may play a decisive role in limiting the triplet formation efficiency in these systems and point more generally to the importance of controlling the hybrid semiconductor interface in applications exploiting exciton-charge carrier interconversion.

\section{EXPERIMENTAL DETAILS}

\section{A. Sample preparation}

Glass substrates $\left(25 \times 25 \mathrm{~mm}^{2}\right.$, VWR $)$ were cleaned sequentially in $2 \%$ Mucasol solution, deionized water, acetone, and isopropanol at $\sim 40{ }^{\circ} \mathrm{C}$ in an ultrasonic bath for $15 \mathrm{~min}$ each, followed by drying under a nitrogen stream. The substrates were UV-ozone treated for $15 \mathrm{~min}$ before perovskite deposition. The perovskite precursor solution was prepared by dissolving methylammonium iodide (1.2M, 99\% Dyenamo) and $\mathrm{PbI}_{2}$ (1.2M, 99.99\% Tokyo Chemical Industry) in a solvent made up of nine parts dimethylformamide (DMF) (Sigma Aldrich, anhydrous, 99.8\%) to one part dimethyl sulfoxide (DMSO) (Sigma Aldrich, anhydrous, 99.9\%). The perovskite precursor solution $(100 \mu \mathrm{l})$ was spin-coated using a two-step spincoating program: $1000 \mathrm{rpm}$ for $10 \mathrm{~s}$ with a ramp-up time of $3 \mathrm{~s}$ and then $4000 \mathrm{rpm}$ for $30 \mathrm{~s}$ with a ramp-up time of $2 \mathrm{~s}$. During the second step, $120 \mu \mathrm{l}$ of antisolvent (chlorobenzene or anisole, Sigma Aldrich, anhydrous, 99.8\%) was dripped at $\mathrm{t}=-5 \mathrm{~s},-10 \mathrm{~s}$, or $-20 \mathrm{~s}$ with respect to the end of the spin-coating program. Finally, the MAPI films were annealed at $100^{\circ} \mathrm{C}$ for $15 \mathrm{~min}$ in nitrogen. The upconversion annihilator layer was deposited by spin coating $100 \mu \mathrm{l}$ of rubrene (Sigma Aldrich, 99.99\%, $10 \mathrm{mg} / \mathrm{ml}$ ): DBP (Sigma Aldrich, $98 \%, 0.1 \mathrm{mg} / \mathrm{ml}$ ) solution in anhydrous chlorobenzene. The spin coating recipe was $6000 \mathrm{rpm}$ for $20 \mathrm{~s}$ with a ramp-up time of $3 \mathrm{~s}$. The bilayer upconversion samples were then left overnight in a nitrogen glove box to dry. The samples were sealed in nitrogen with a microscope glass coverslip using Blufixx epoxy and a UV light.

\section{B. Photoluminescence measurements}

The laser source was a NKT SuperK Fianium supercontinuum laser, with an adjustable repetition rate and user-selectable output wavelength band (Varia). Samples were measured using a homebuilt inverted confocal fluorescence microscope with a computercontrolled piezoscanner stage using the QuDI software suite. ${ }^{37}$ Excitation and detection were through the annihilator layer in confocal configuration using a low-magnification air objective and no imaging pinhole. Photoluminescence detection was via a silicon single-photon avalanche diode (Excelitas SPCM-AQRH-14) for PL mapping and time-correlated photon-counting measurements and via a silicon CCD-backed spectrograph (Teledyne Princeton Instruments Isoplane 160, PIXIS:100B eXcelon) for PL spectra. The optical path could be reproducibly toggled between the two detectors using a motorized swing mirror without changing the optical filtering or background conditions. For time-correlated photon-counting measurements, we used a TimeHarp 260 Nano (Picoquant). The laser spot size was calculated from a measurement of laser scatter mapped across the sharply defined edge of a cleaved silicon wafer, positioned in the sample plane. Modeling the laser spot as a Gaussian of the form $\mathrm{I}(x) \propto \exp \left(-\left(x-x_{0}\right)^{2} /\left(d^{2}\right)\right)$, the diameter $d$ was $8.6 \mu \mathrm{m}$. Laser power was adjusted using a variable neutral density filter and measured from a fixed reference position using a power meter (Thorlabs PM100-D) and a calibrated silicon photodiode.

The use of a supercontinuum laser entails a small but potentially significant broadband laser signal in the excitation channel. For this reason, copious optical filtering was applied during all measurements. All filters were Thorlabs Premium grade except for the $715 \mathrm{~nm}$ longpass filter (Semrock, SEM-FF01-715/LP-25). MAPI photoluminescence measurements used an excitation band of $670 \mathrm{~nm}-680 \mathrm{~nm}$, two shortpass filters on the excitation source (700 $\mathrm{nm}$ and $750 \mathrm{~nm}$ ), and two longpass filters on the detector (715 $\mathrm{nm}$ and $750 \mathrm{~nm})$. Upconversion measurements used an excitation band of $715 \mathrm{~nm}-750 \mathrm{~nm}$, two longpass filters on the excitation source $(650 \mathrm{~nm}$ and $715 \mathrm{~nm})$, and two shortpass filters on the detector $(700 \mathrm{~nm}$ and $650 \mathrm{~nm})$. During time-resolved upconversion measurements, an additional $600 \mathrm{~nm}$ shortpass filter was applied to the detector to fully remove any MAPI PL signal. Directly excited annihilator measurements used an excitation band of $425 \mathrm{~nm}-435 \mathrm{~nm}$, a $450 \mathrm{~nm}$ shortpass filter on the laser, and a $500 \mathrm{~nm}$ longpass filter on the detector. Quasi-continuous wave measurements utilized a laser repetition rate of $78.2 \mathrm{MHz}$.

\section{RESULTS AND DISCUSSION}

\section{A. MAPI PL maps}

We varied the spin-coating procedure followed during the perovskite film formation, before depositing an identical rubrene:DBP 
layer over each film. Scanning electron microscope (SEM) images were collected for each MAPI film variety and are shown in Fig. S1 of the supplementary material. The film thickness was approximately $500 \mathrm{~nm}$ in all cases. The antisolvent drip time had a large effect on the crystallite size and pinhole density, which both diminished with increasing drip time. Films formed at $5 \mathrm{~s}$ and $10 \mathrm{~s}$ drip times contained visible voids in the film structure, possibly as a result of poor packing of the very large crystals. Note that we define the dripping
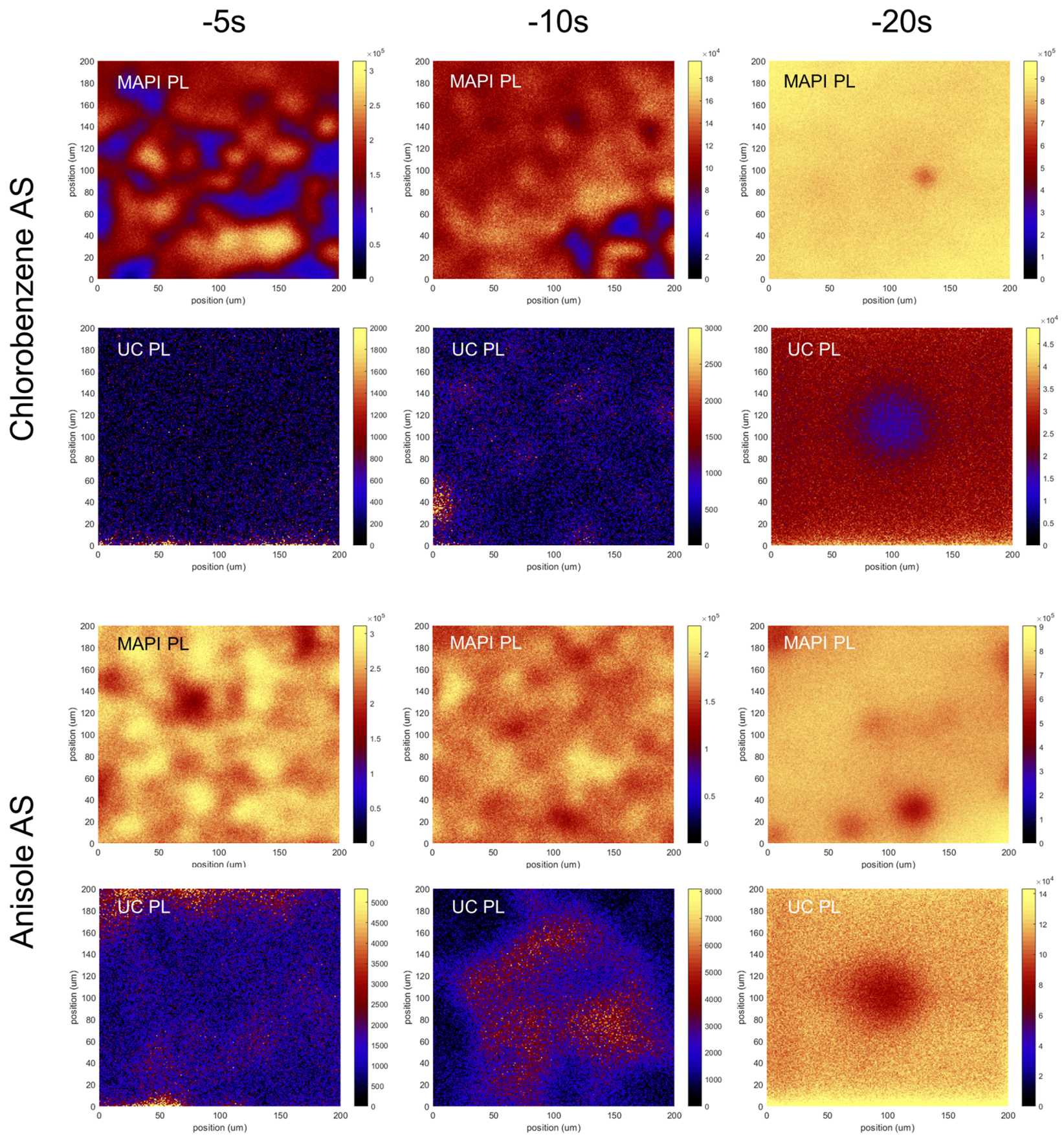

FIG. 2. Spatially resolved $200 \times 200 \mu \mathrm{m}^{2}$ maps of MAPI photoluminescence (PL) and TTA-upconversion PL collected for anisole- and chlorobenzene antisolvent (AS)-treated MAPI with identically prepared rubrene:DBP annihilator layers, with three different antisolvent drip times utilized during MAPI film formation. The absolute intensities and the uniformity are greatest for the $20 \mathrm{~s}$ antisolvent drip time. Darker spots in the center of the UC images are the result of photodegradation, which is accelerated in the middle of the image due to the laser parking at this position between measurements. 
time in units of seconds prior to the end of the spin-coating program; hence, for the $20 \mathrm{~s}$ drip time, the antisolvent is applied at the earliest. The choice of antisolvent-anisole or chlorobenzene-did not have a noticeable effect on the film characteristics observable in SEM.

Spatial maps of the photoluminescence (PL) signals from MAPI band-to-band recombination and from TTA-upconversion in the annihilator layer were collected over a fixed $200 \times 200 \mu \mathrm{m}^{2}$ area for a series of excitation densities. We verified that the signal collected by the avalanche photodiode was made up of the expected PL spectrum and was free from background and laser signals by periodically collecting PL spectra throughout the experiment. Representative PL spectra are shown in Fig. S2. The MAPI and upconversion PL maps collected at the highest excitation intensity, $900 \mathrm{~W} / \mathrm{cm}^{2}$, are displayed in Fig. 2. The spatially integrated intensities for each excitation density and perovskite film composition are displayed in Fig. 3.

The uniformity and brightness of the MAPI PL both increased as the antisolvent dripping time was increased from $5 \mathrm{~s}$ to $10 \mathrm{~s}$ to $20 \mathrm{~s}$. This agreed with our expectations: a time in the vicinity of $20 \mathrm{~s}$ is common in recipes for lead halide perovskite-based photovoltaics, and PL uniformity and brightness are both indicators of a film capable of producing a high-efficiency device with minimal defect-related open-circuit voltage losses. ${ }^{19}$ The spatial variation of the MAPI PL from the $5 \mathrm{~s}$ and $10 \mathrm{~s}$ samples is likely associated with the abundance of voids and pinholes observed for these samples by using SEM. We did not observe a substantial difference in the MAPI $\mathrm{PL}$ intensity when switching between the two antisolvents, which is also consistent with the SEM result.

Occasional darker regions are visible in the $20 \mathrm{~s}$ MAPI PL maps. These were confirmed through sequential measurements to be intrinsic to the film and not generated by photodegradation. These regions may represent the locations of particularly effective defects, and their appearance suggests that the film processing may yet be improved slightly.

The MAPI PL intensities are plotted against excitation intensity on double logarithmic axes in Fig. 3. The response of each sample follows a straight line, indicating adherence to the relation $\mathrm{I}_{\mathrm{pl}} \propto \mathrm{I}_{\text {exc }}{ }^{\mathrm{k}}$, where $\mathrm{k}$ is a constant denoting the radiative ideality factor. ${ }^{38}$ The value of $\mathrm{k}$ is $\sim 1.65$ for the less-bright films and 1.51 for the brightest films, formed with the $20 \mathrm{~s}$ dripping time. Ideal bandto-band recombination should yield a $\mathrm{k}$ of 1.0 in the high-injection limit, and the deviations seen here may indicate the involvement of bulk defect states in the radiative recombination process. The reduction of $\mathrm{k}$ would then indicate a material bulk with a lower bulk defect density, consistent with the higher photoluminescence yield.

\section{B. Upconversion PL maps}

The response of the upconversion PL maps to antisolvent drip time followed those of the MAPI PL, as depicted in Fig. 2. As the antisolvent dripping time increased, so too did the intensity and uniformity of the upconversion signal. Dark spots in the middle of the upconversion PL maps and bright fringes at the image edges are the results of photodegradation, as discussed below.

The upconversion PL intensities plotted against excitation intensity in Fig. 3 give an impression of the relative brightness of each system as well as the TTA efficiency. While the choice of antisolvent had little systematic effect on the MAPI photoluminescence intensity, it had a surprisingly strong influence on the upconversion intensity. For all three dripping times, the anisole system produced an upconversion intensity $\sim 10$ times greater than that of the chlorobenzene, depending on the excitation intensity. We measured the upconversion efficiency of the two brightest samples using the methodology detailed in Sec. 3 of the supplementary material; the excitation intensity-dependent efficiencies are plotted in Fig. S3.
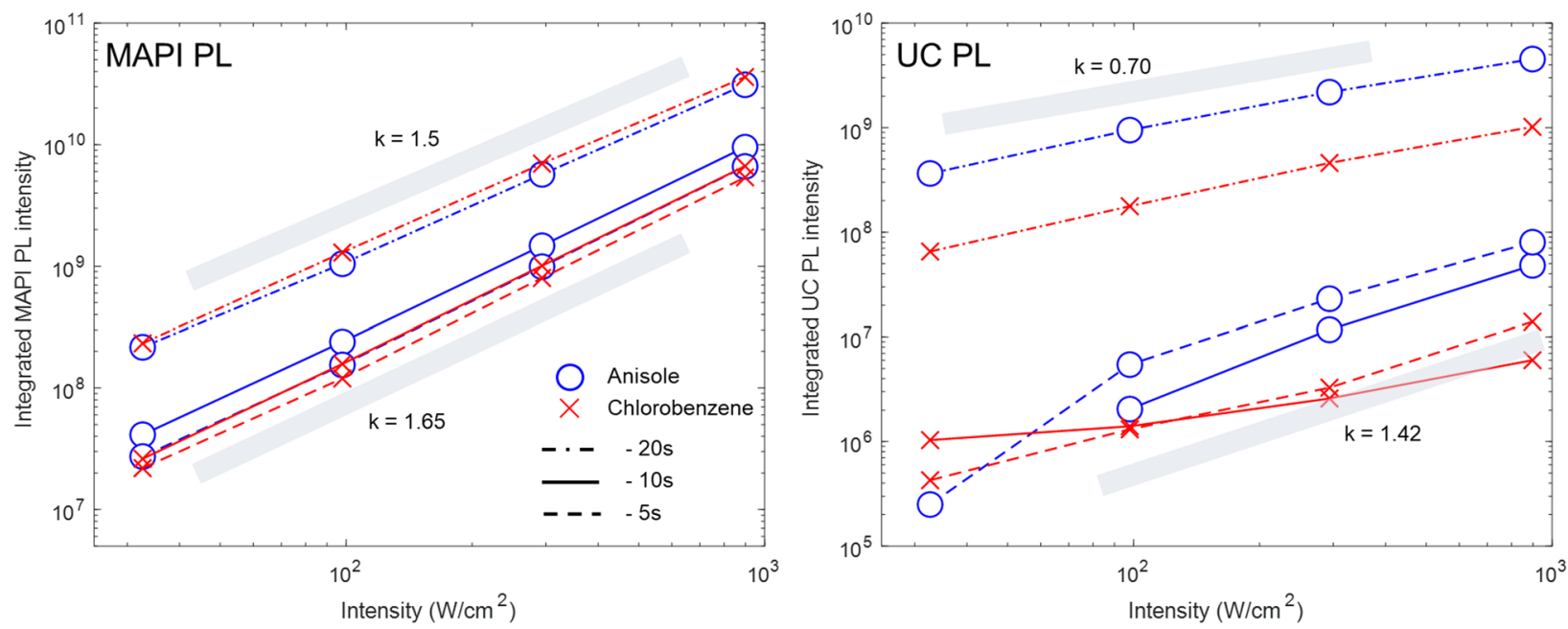

FIG. 3. Double logarithmic plots of the spatially integrated MAPI (left) and UC (right) PL intensities for each film formation condition against excitation intensity. The gray lines are indicative of the stated value of $\mathrm{k}$, where $\mathrm{k}$ is the exponent in the excitation dependence of the photoluminescence intensity. 
The maximum values for the brightest anisole- and chlorobenzenetreated samples were $0.073 \%$ and $0.006 \%$, respectively. Our investigation into the origin of this unexpected difference in efficiency is below.

The TTA process is one of the several channels by which tripletexcited rubrene can relax, and since TTA is bimolecular, the likelihood of triplets participating in TTA is triplet density-dependent, hence the classic relationship whereby upconversion intensity follows the form $\mathrm{I}_{\mathrm{UC}} \propto[\mathrm{T}]^{\mathrm{k}([\mathrm{T}])}$, where $[\mathrm{T}]$ is the triplet density and $\mathrm{k}$ varies from 2 to 1 as $[\mathrm{T}]$ increases, signifying a transition from inefficient to efficient TTA. ${ }^{39}$ The slope of the intensity response in Fig. 3 is equal to $\mathrm{k}$.

For the least-bright upconverters plotted in Fig. 3, k averages 1.42 , signifying that the triplet density in the annihilator is too low to support efficient TTA. Inefficient triplet generation at the MAPI/rubrene interface is likely the source of this. For the two brightest upconverters, produced using $t=20 \mathrm{~s}$ drip time, the value of $\mathrm{k}$ is 0.70 across the excitation intensity range measured. We interpret this value of $\mathrm{k}$ to mean that the system has met the criteria for efficient TTA in the annihilator layer, while also being subjected to another carrier density-dependent process that reduces the overall upconversion efficiency. Assuming an identical optical system, the intensity difference between the two upconverters in the efficient TTA regime is directly relatable to the triplet density, so this result suggests that the carrier-driven triplet formation in the anisole sample is roughly ten times more efficient than in the chlorobenzene sample.

A prime candidate for the second density-dependent process, which diminishes the upconversion efficiency as the excitation density increases, is carrier recombination in the MAPI bulk. The carrier recombination rate in MAPI increases superlinearly with carrier density as band-to-band and ultimately Auger recombination mechanisms come to dominate carrier decay. ${ }^{40}$ The effect of this accelerating recombination rate is a decrease in the carrier diffusion length, leading to a decoupling of the surface from the bulk and a reduced probability per absorbed photon of generating triplets at the MAPI/rubrene interface. Engineering a system where the interface-related triplet generation process remains operational at high perovskite carrier densities may prove to be a challenge going forward.

\section{MAPI carrier lifetime measurement}

Searching for a clue that may explain the different upconversion performance of the anisole- and chlorobenzene-treated $20 \mathrm{~s}$ bilayers, we examined the two samples using UV-vis-NIR spectrophotometry (Fig. S4) and surface photovoltage measurements (Fig. S5). Considered alongside the results of the quasi-continuous wave photoluminescence (Fig. 2) and SEM (Fig. S1) measurements, it is clear that with the exception of the upconversion PL intensity, the two samples are virtually indistinguishable. What then could be responsible for the different upconversion intensities?

Time-resolved photoluminescence measurements of the MAPI films provided an answer. As shown in Fig. 4, we measured MAPI PL transients for the two bilayer samples at a range of excitation fluences using a laser repetition rate of $151 \mathrm{kHz}$. Our objective was to measure the nonradiative recombination-limited carrier lifetime of the two samples, which manifests under low carrier density conditions such that nonradiative recombination becomes the main lifetimelimiting process. ${ }^{40}$ We assume that the MAPI PL transients collected at the lowest fluence, $45 \mathrm{~nJ} / \mathrm{cm}^{2}$, are reflective of this regime. These low-fluence transients were fitted with a single exponential decay to extract the nonradiative recombination-limited carrier lifetimes.

The MAPI PL transients differ substantially between the two samples. The carrier lifetime in the anisole-treated sample is $306 \mathrm{~ns}$, compared to $52 \mathrm{~ns}$ for the chlorobenzene-treated sample. What could be the source of this diminished carrier lifetime? It is noteworthy that the PL intensity of the two samples measured under quasi-continuous wave conditions was near-identical, differing by
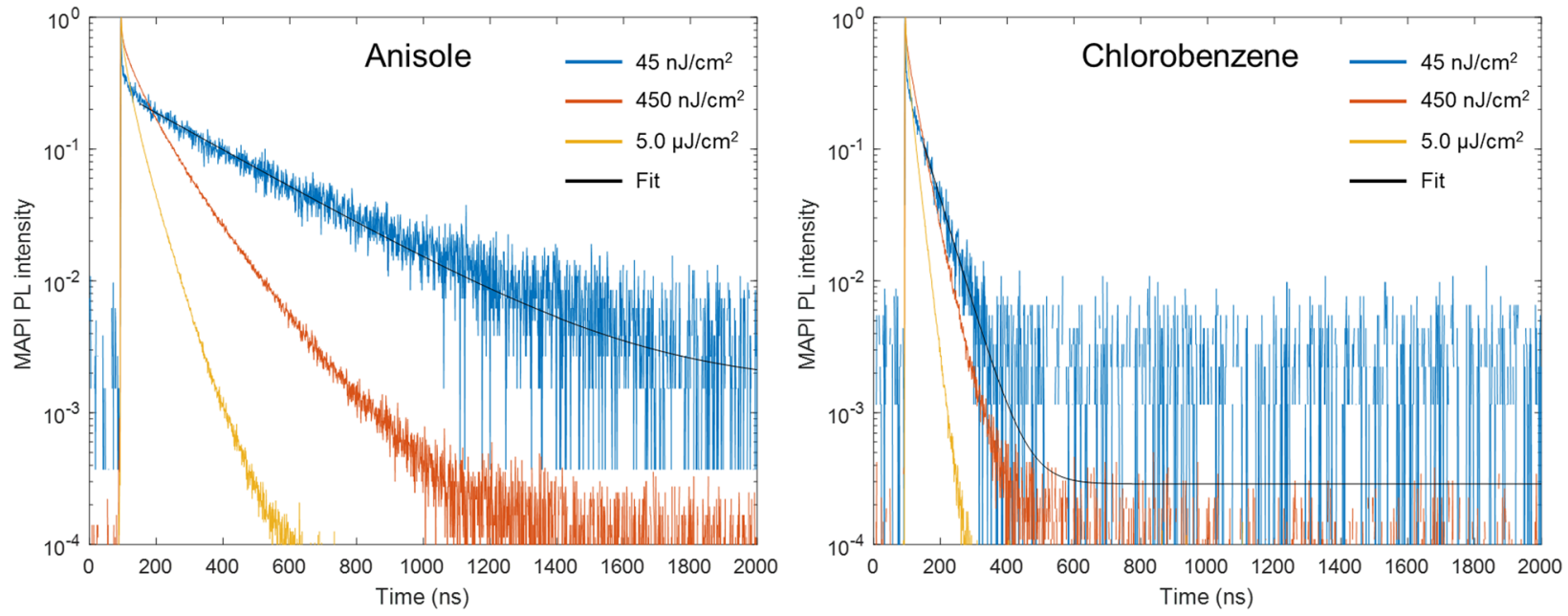

FIG. 4. MAPI PL transients for anisole- and chlorobenzene-treated MAPI films with rubrene:DBP annihilator layers. 
$\sim 20 \%$ in favor of the chlorobenzene-treated sample, as shown in Figs. 2 and 3. Since MAPI band-to-band PL is a bulk effect, we take this to mean that the bulk of both materials have near-identical properties. This conclusion is reinforced by the indistinguishability of both samples in spectrophotometry, surface photovoltage, and SEM measurements.

Given the similarity of the bulk in both samples, the lower carrier lifetime of the chlorobenzene sample may be the result of an increased defect density at the MAPI/rubrene interface. This interpretation is consistent with the antisolvent treatment having a large effect on the upconversion performance without appreciably modifying any aspects of the bulk MAPI or annihilator layer properties. The process by which MAPI carriers generate triplet excitons in the rubrene:DBP layer has not yet been definitively identified. However, the process must be mediated by the MAPI/rubrene interface and is presumably sensitive to defects there.

The nonradiative recombination-limited carrier lifetime of the two samples differs by a factor of $\sim 6$, which is similar to the difference in the upconversion intensity of the two films. A linear dependence between carrier lifetime and upconversion intensity is consistent with the slope of the excitation intensity-dependent upconversion response depicted in Fig. 3. Our analysis of the value $k=0.70$, derived from fitting the double logarithmic plot of the intensitydependent upconversion response, concluded that TTA was efficient in both systems across the range of excitation intensities supplied. Any increase in triplet density while in this regime should produce an approximately linear increase in the upconversion intensity. The longer carrier lifetime in the anisole-treated film is therefore presumably directly connected to a more efficient triplet generation process.

\section{Upconversion impulsive response measurement}

An analysis of the transient response of the upconversion photoluminescence supports the conclusion that a longer MAPI carrier lifetime coincides with more efficient triplet generation. Operating the laser at a repetition rate of $3.125 \mathrm{MHz}$, we collected photoluminescence transients over a time window of $320 \mathrm{~ns}$. The normalized transients are plotted in Fig. 5. The measurement window was deliberately selected to be much shorter than the rubrene triplet lifetime, which is of the order tens of microseconds. This measurement configuration enabled us to see the response of the system under excitation conditions that more closely resembled the steadystate, in which TTA is occurring efficiently throughout the measurement window. The upconversion "transient" therefore consisted of a steady-state component superimposed with the transient response of the system to a single short laser pulse.

The rise of the impulsive response signal was fitted with a simple model, $\mathrm{A}\left[1-\exp \left(-\left(\mathrm{t}-\mathrm{t}_{0}\right) / \tau_{\text {rise }}\right)\right]$, where $\tau_{\text {rise }}$ is the rise time, $\mathrm{t}_{0}$ is the experiment time-zero, and $\mathrm{A}$ is the amplitude. The rise time of the upconversion signal is $14 \mathrm{~ns}$ in the anisole-treated sample and $6 \mathrm{~ns}$ in the chlorobenzene-treated sample. The magnitude of the rise in the upconversion signal in response to the laser pulse is four times greater in the anisole-treated sample.

The longer rise time of the anisole-treated sample suggests that triplet generation occurs for a longer time following the laser pulse. Likewise, the greater magnitude of the impulsive response indicates a larger quantity of triplets being formed per laser pulse.

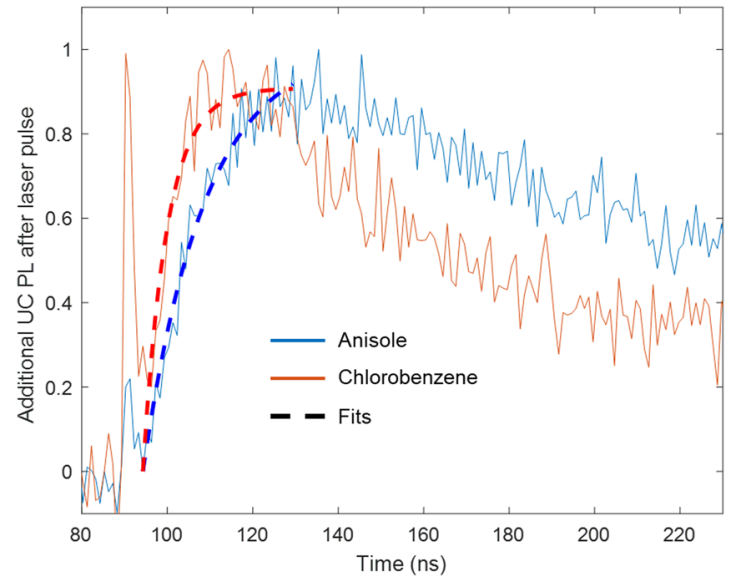

FIG. 5. Upconversion PL transients of the two 20 s MAPI/rubrene:DBP films, measured in a short time window such that a steady background triplet concentration was sustained and the impulsive response to a laser pulse is superimposed. Fits to each transient indicate the upconversion signal rise time. The magnitude of the anisole-treated film response was four times greater than that of the chlorobenzene-treated film. The spike at $90 \mathrm{~ns}$ is a result of scattered laser light in the detector channel.

The diffusion of triplets away from the MAPI/rubrene interface and the impact of this on the time-dependent annihilation probability mean that deriving a more quantitative insight from these transients requires numerical simulation. However, the results broadly support the argument that the surface defect profile at the MAPI/rubrene interface, as indicated by the carrier lifetime, plays a central role in triplet formation.

\section{E. Upconverter photodegradation dynamics}

We observed a rapid light-induced degradation of the upconversion signal, similar to that reported by Wieghold and Nienhaus. ${ }^{29}$ The effect is apparent in the brighter upconversion maps in Fig. 2, appearing as a dark spot in the center of each map. The photodegradation effect was strongest in the center of each image as the laser beam was parked in this position in between the collection of maps at different excitation densities. A stronger upconversion signal detected near the edges of some maps is similarly a result of these areas experiencing less cumulative laser exposure.

The degradation kinetics of the two $20 \mathrm{~s}$ samples were measured by collecting a continuous sequence of short-exposure time upconversion PL spectra over a 10 min period using the CCD spectrograph, beginning the measurement coincident with the exposure of a fresh region of the sample to the laser beam. No significant change in the shape of the PL spectrum was seen during the measurement; the decay in intensity affected all portions of the spectrum equally. This suggests that the degradation of the upconversion signal was not caused by the changes in the bulk of the annihilator layer, which would affect the shape of the delayed PL spectrum.

The spectrally integrated intensity plots for the two samples are found in Fig. 6. The relative decay in upconversion intensity over the $10 \mathrm{~min}$ measurement window is shown. The decay transients exhibit obvious faster and slower-decaying regimes. The 


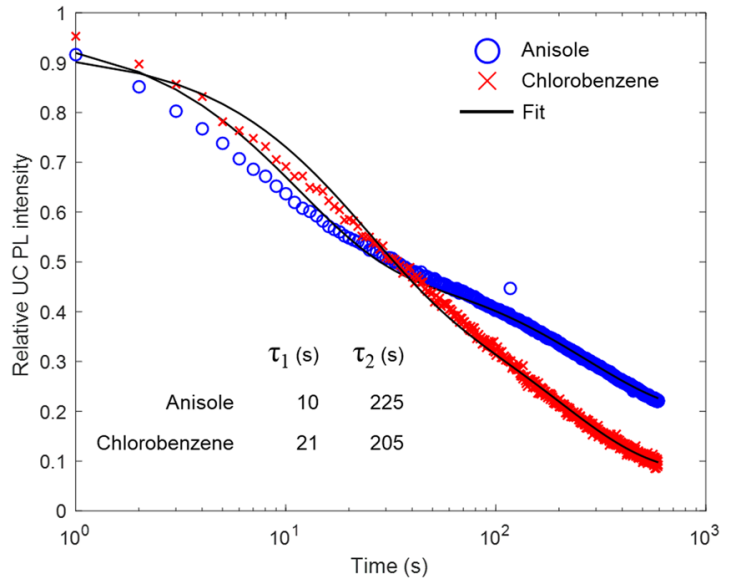

FIG. 6. Relative decay in the upconversion intensity of the two upconverters measured with a fixed sample position and a quasi-continuous wave excitation intensity of $40 \mathrm{~W} / \mathrm{cm}^{2}$. The decay transients were fitted with a biexponential decay model. The fit parameters are given in the inset table.

transients were fitted with a biexponential model. The upconversion signal from the anisole-treated sample decayed more rapidly than the chlorobenzene equivalent, with a fast component of $10 \mathrm{~s}$ compared to $21 \mathrm{~s}$, while the slower decay component was similar$\sim 210 \mathrm{~s}$-for both samples. While we did not rigorously quantify the excitation intensity-dependence of the upconversion signal decay, the decay rate did seem to increase at higher laser powers. The excitation intensity used for this measurement, $40 \mathrm{~W} / \mathrm{cm}^{2}$, was selected as a compromise between giving sufficient signal per unit time and a degradation rate slow enough to be resolvable using the CCD spectrograph measurement.

The observation of a decay in upconversion PL with two clear components on the timescale of seconds is consistent with the precharging mechanism reported by Wieghold and Nienhaus. ${ }^{29}$ On the timescale of minutes, we did not see a recovery of the photobleaching effect after resting the sample in the dark. We were unable to check for a yet-slower recovery of the photobleach effect, on the timescale of hours, due to the limited lifespan of the sample encapsulation.

We did not observe any decay in the MAPI PL that correlated with the diminishing upconversion signal. Since MAPI PL is a bulk phenomenon and appeared unconnected to the degradation of the upconversion signal, this suggests that the degradation was specific to some aspect of the MAPI/rubrene interface. The different decay rates of the anisole- and chlorobenzene-treated samples support this. Our bulk-sensitive measurements showed that the two samples were effectively identical except in the matter of carrier lifetime. The interface is the logical answer to the question of what aspect of the two samples must differ.

\section{CONCLUSIONS}

We found that TTA-upconversion was generated in bilayer MAPI/rubrene:DBP samples, with the intensity of the upconversion signal strongly dependent on the characteristics of the underlying
MAPI. MAPI films that exhibited characteristics in line with efficient photovoltaic cell performance-a closed layer and bright and uniform photoluminescence-were produced with an antisolvent dripping time of $20 \mathrm{~s}$ with respect to the end of a $40 \mathrm{~s}$ spin-coating program. These films produced the brightest upconverters and exhibited an excitation intensity response that suggested that efficient TTA was occurring within the annihilator layer, despite the low overall efficiency of the upconversion process.

The antisolvent had a large effect on the upconversion efficiency, with anisole producing a roughly tenfold increase over chlorobenzene. We attribute this difference to a more efficient triplet generation process operating in the anisole-treated sample. A hint as to the possible origin of this difference in triplet generation efficiency is the MAPI carrier lifetime, which differed by a factor of 6 between the brightest anisole- and chlorobenzene-treated samples. Given the close similarity in the bulk properties of the two materials, as determined by a range of bulk-sensitive measurements, we attributed the different carrier lifetimes to a changing defect density on the MAPI surface induced during antisolvent processing.

It is reasonable to suppose that the differences in the MAPI carrier lifetime, rubrene triplet formation efficiency, and upconversion photodegradation rate may have a common source, related to the MAPI/rubrene interface and the local defect density. Further investigation is necessary to confirm these connections and to identify the underlying causative factors. By understanding the internal processes that limit triplet formation in perovskite-sensitized TTA upconverters, it may be possible to circumvent them and follow the remarkable performance rise seen in other halide perovskite-based optoelectronics.

\section{SUPPLEMENTARY MATERIAL}

The supplementary material contains SEM images, photoluminescence spectra, a derivation of the photon upconversion efficiency, absorbance spectra, and surface photovoltage spectra.

\section{AUTHORS' CONTRIBUTIONS}

K.P. prepared samples, performed measurements, and analyzed data. B.N. developed and configured the photoluminescence setup. K.L. and E.U. contributed to planning and analysis. R.W.M. conceived this study, performed measurements, analyzed data, and prepared this manuscript. All authors contributed to and commented on this manuscript.

\section{ACKNOWLEDGMENTS}

K.P. thanks the Deutscher Akademischer Austauschdienst (DAAD) for funding via the Research Grants-Doctoral Programmes in Germany. B.N. acknowledges financial support from the DFG (Project Nos. 410866378 and 410866565). E.U. acknowledges funding from the Bundesministerium für Bildung und Forschung (BMBF). R.W.M. acknowledges the Helmholtz Association, Germany, for funding within the Helmholtz Excellence Network SOLARMATH, a strategic collaboration of the DFG Excellence Cluster MATH+ and Helmholtz-Zentrum Berlin (Grant No. ExNet-0042-Phase-2-3). This work was partially supported by the 
BMBF through the collaborative projects EPRoC (Contract No. 01186916/1) and SISSY (Contract No. 03SF0403) as well as the Helmholtz Energy Materials Foundry (HEMF) funded by the Helmholtz Association (HGF). All authors are grateful to Carola Klimm (HZB) for SEM measurements and PD Dr. Thomas Dittrich (HZB) for support with surface photovoltage measurements and discussions.

\section{DATA AVAILABILITY}

The data that support the findings of this study are available from the corresponding author upon reasonable request.

\section{REFERENCES}

${ }^{1}$ S. Wen et al., "Future and challenges for hybrid upconversion nanosystems," Nat. Photonics 13(12), 828-838 (2019).

${ }^{2}$ B. D. Ravetz et al., "Photoredox catalysis using infrared light via triplet fusion upconversion," Nature 565(7739), 343-346 (2019).

${ }^{3}$ S. H. C. Askes and S. Bonnet, "Solving the oxygen sensitivity of sensitized photon upconversion in life science applications," Nat. Rev. Chem. 2, 437 (2018).

${ }^{4}$ T. F. Schulze and T. W. Schmidt, "Photochemical upconversion: Present status and prospects for its application to solar energy conversion," Energy Environ. Sci. 8(1), 103-125 (2015).

${ }^{5} \mathrm{~L}$. Nienhaus et al., "Triplet-sensitization by lead halide perovskite thin films for near-infrared-to-visible upconversion," ACS Energy Lett. 4, 888-895 (2019).

${ }^{6} \mathrm{~N}$. Yanai et al., "Increased vis-to-UV upconversion performance by energy level matching between a TADF donor and high triplet energy acceptors," J. Mater. Chem. C 4, 6447 (2016).

${ }^{7}$ T. C. Wu, D. N. Congreve, and M. A. Baldo, "Solid state photon upconversion utilizing thermally activated delayed fluorescence molecules as triplet sensitizer," Appl. Phys. Lett. 107(3), 031103 (2015).

${ }^{8} \mathrm{M}$. Wu et al., "Solid-state infrared-to-visible upconversion sensitized by colloidal nanocrystals," Nat. Photonics 10(1), 31-34 (2016).

${ }^{9} \mathrm{~K}$. Okumura et al., "Employing core-shell quantum dots as triplet sensitizers for photon upconversion," Chem. - Eur. J. 22, 7721 (2016).

${ }^{10}$ S. Garakyaraghi and F. N. Castellano, "Nanocrystals for triplet sensitization: Molecular behavior from quantum-confined materials," Inorg. Chem. 57(5), 2351-2359 (2018)

${ }^{11}$ L. Nienhaus et al., "Speed limit for triplet-exciton transfer in solid-state $\mathrm{PbS}$ nanocrystal-sensitized photon upconversion," ACS Nano 11(8), 7848-7857 (2017).

${ }^{12} \mathrm{P}$. Xia et al., "Achieving spin-triplet exciton transfer between silicon and molecular acceptors for photon upconversion," Nat. Chem. 12(2), 137-144 (2020).

${ }^{13} \mathrm{~K}$. Mase et al., "Triplet sensitization by perovskite nanocrystals for photon upconversion," Chem. Commun. 53, 8261 (2017).

${ }^{14} \mathrm{~S}$. Amemori et al., "Near-infrared-to-visible photon upconversion sensitized by a metal complex with spin-forbidden yet strong $\mathrm{S}_{0}-\mathrm{T}_{1}$ absorption," J. Am. Chem. Soc. 138(28), 8702-8705 (2016).

${ }^{15} \mathrm{~W}$. Wu et al., "Organic triplet sensitizer library derived from a single chromophore (BODIPY) with long-lived triplet excited state for triplet-triplet annihilation based upconversion," J. Org. Chem. 76(17), 7056-7064 (2011).

${ }^{16} \mathrm{R}$. Wang et al., "Constructive molecular configurations for surface-defect passivation of perovskite photovoltaics," Science 366(6472), 1509-1513 (2019).

${ }^{17}$ I. Zarazua et al., "Surface recombination and collection efficiency in perovskite solar cells from impedance analysis," J. Phys. Chem. Lett. 7(24), 5105-5113 (2016).

${ }^{18} \mathrm{Y}$. Yang et al., "Top and bottom surfaces limit carrier lifetime in lead iodide perovskite films," Nat. Energy 2, 16207 (2017).
${ }^{19} \mathrm{M}$. Stolterfoht et al., "Visualization and suppression of interfacial recombination for high-efficiency large-area pin perovskite solar cells," Nat. Energy 3(10), 847-854 (2018)

${ }^{20}$ T. H. Han et al., "Interface and defect engineering for metal halide perovskite optoelectronic devices," Adv. Mater. 31(47), 1803515 (2019).

${ }^{21}$ S. Cong et al., "Organic small molecule as the underlayer toward high performance planar perovskite solar cells," ACS Appl. Mater. Interfaces 9(3), 2295-2300 (2017).

${ }^{22} \mathrm{G}$. Ji et al., "Interfacial electronic structures revealed at the rubrene/ $/ \mathrm{CH}_{3} \mathrm{NH}_{3} \mathrm{PbI}_{3}$ interface," Phys. Chem. Chem. Phys. 19(9), 6546-6553 (2017).

${ }^{23} \mathrm{H}$. Lim et al., "Delayed triplet-state formation through hybrid charge transfer exciton at copper phthalocyanine/GaAs heterojunction," J. Phys. Chem. Lett. 8(19), 4763-4768 (2017).

${ }^{24} \mathrm{R}$. Younts et al., "Efficient generation of long-lived triplet excitons in 2D hybrid perovskite," Adv. Mater. 29(9), 1604278 (2017).

${ }^{25} \mathrm{~K}$. Ema et al., "Nearly perfect triplet-triplet energy transfer from Wannier excitons to naphthalene in organic-inorganic hybrid quantum-well materials," Phys. Rev. Lett. 100(25), 257401 (2008).

${ }^{26} \mathrm{M}$. Braun et al., "Tailoring of energy levels in lead chloride based layered perovskites and energy transfer between the organic and inorganic planes," Chem. Phys. Lett. 303(1), 157-164 (1999).

${ }^{27}$ J. S. Manser, J. A. Christians, and P. V. Kamat, "Intriguing optoelectronic properties of metal halide perovskites," Chem. Rev. 116(21), 12956-13008 (2016).

${ }^{28} \mathrm{~S}$. Wieghold et al., "Triplet sensitization by lead halide perovskite thin films for efficient solid-state photon upconversion at subsolar fluxes," Matter 1(3), 705-719 (2019).

${ }^{29} \mathrm{~S}$. Wieghold and L. Nienhaus, "Precharging photon upconversion: Interfacial interactions in solution-processed perovskite upconversion devices," J. Phys. Chem. Lett. 11(3), 601-607 (2020).

${ }^{30} \mathrm{~S}$. Wieghold et al., "Is disorder beneficial in perovskite-sensitized solid-state upconversion? The role of DBP doping in rubrene," J. Phys. Chem. C 124, 18132 (2020).

${ }^{31}$ A. S. Bieber et al., "Perovskite-sensitized upconversion bingo: Stoichiometry, composition, solvent, or temperature?," J. Chem. Phys. 153(8), 084703 (2020).

${ }^{32} \mathrm{~S}$. Wieghold et al., "One-step fabrication of perovskite-based upconversion devices," ChemPhotoChem 4(9), 704-712 (2020).

${ }^{33} \mathrm{~J}$. Lim et al., "Elucidating the long-range charge carrier mobility in metal halide perovskite thin films," Energy Environ. Sci. 12(1), 169-176 (2019).

${ }^{34} \mathrm{P}$. Zhao et al., "Antisolvent with an ultrawide processing window for the onestep fabrication of efficient and large-area perovskite solar cells," Adv. Mater. 30(49), 1802763 (2018).

${ }^{35} \mathrm{D}$. Prochowicz et al., "Understanding the effect of chlorobenzene and isopropanol anti-solvent treatments on the recombination and interfacial charge accumulation in efficient planar perovskite solar cells," J. Mater. Chem. A 6(29), 14307-14314 (2018).

${ }^{36}$ T.-B. Song et al., "Dynamics of antisolvent processed hybrid metal halide perovskites studied by in situ photoluminescence and its influence on optoelectronic properties," ACS Appl. Energy Mater. 3(3), 2386-2393 (2020).

${ }^{37} \mathrm{~J}$. M. Binder et al., "Qudi: A modular python suite for experiment control and data processing," SoftwareX 6, 85-90 (2017).

${ }^{38}$ T. Schmidt, K. Lischka, and W. Zulehner, "Excitation-power dependence of the near-band-edge photoluminescence of semiconductors," Phys. Rev. B 45(16), 8989-8994 (1992).

${ }^{39}$ A. Monguzzi et al., "Low power, non-coherent sensitized photon upconversion: Modelling and perspectives," Phys. Chem. Chem. Phys. 14(13), 43224332 (2012).

${ }^{40}$ S. D. Stranks, "Nonradiative losses in metal halide perovskites," ACS Energy Lett. 2(7), 1515-1525 (2017). 